\title{
Binaries and distances
}

\author{
D. Pourbaix, ${ }^{1,2}$ F. Arenou, ${ }^{3}$ J.-L. Halbwachs,${ }^{4}$ and C. Siopis ${ }^{2}$ \\ ${ }^{1}$ F.R.S.-FNRS, 1000 Brussels, Belgium \\ email: pourbaix@astro.ulb.ac.be \\ ${ }^{2}$ Institut d'Astronomie et d'Astrophysique, Université Libre de Bruxelles (ULB), CP 226, \\ Bld. du Triomphe, 1050 Brussels, Belgium \\ ${ }^{3}$ GEPI, Observatoire de Paris, CNRS, 92195 Meudon, France \\ ${ }^{4}$ Observatoire Astronomique de Strasbourg, UMR 7550, 67000 Strasbourg, France
}

\begin{abstract}
Gaia's five-year observation baseline might naively lead to the expectation that it will be possible to fit the parallax of any sufficiently nearby object with the default five-parameter model (position at a reference epoch, parallax and proper motion). However, simulated Gaia observations of a 'model Universe' composed of nearly $10^{7}$ objects, $50 \%$ of which turn out to be multiple stars, show that the single-star hypothesis can severely affect parallax estimation and that more sophisticated models must be adopted. In principle, screening these spurious single-star solutions is rather straightforward, for example by evaluating the quality of the fits. However, the simulated Gaia observations also reveal that some seemingly acceptable single-star solutions can nonetheless lead to erroneous distances. These solutions turn out to be binaries with an orbital period close to one year. Without auxiliary (e.g., spectroscopic) data, they will remain unnoticed.
\end{abstract}

Keywords. astrometry, binaries: general, stars: distances, Cepheids

\section{Introduction}

In an ideal world composed of single stars only, the quasi-periodic motion of the photocenter of an object would be directly related to its parallax, and the displacement over one year would coincide with its annual proper motion. However, the world is not that ideal and both the parallax and proper motion derived assuming that an object is a single star can be severely biased for binary or multiple systems. This is even more worrisome when the binary object turns out to be one of the few accessible specimens of a class of standard candles such as Cepheids.

\section{Confirmed effect for known binaries}

The effect of binarity on the fitted parallax was illustrated by Pourbaix \& Jorissen (2000) for a sample of $\mathrm{Ba}, \mathrm{CH}$ and $\mathrm{S}$ stars (see Fig. 1, left). They showed that the effect is maximal for orbital periods close to one year, based on Hipparcos observations. However, right at the time of release of the Hipparcos catalogue (ESA 1997), Szabados (1997) already expressed some concerns about the parallax of binary Cepheids for which the single-star model was used to fit the Hipparcos observations. At that time, there were 19 known binary Cepheids out to $1 \mathrm{kpc}$. To date, 56 Cepheids have been identified in the Hipparcos Catalogue out to that distance (see Fig 1, right), 40 of which are already known to be binaries. Fortunately, among those with a known orbit, only Z Lac has an orbital period close to $1 \mathrm{yr}$.

In the context of the Hipparcos observing campaign, the target objects were known in advance and so was their binary status. In the case of a blind analysis (e.g., as for Gaia), 

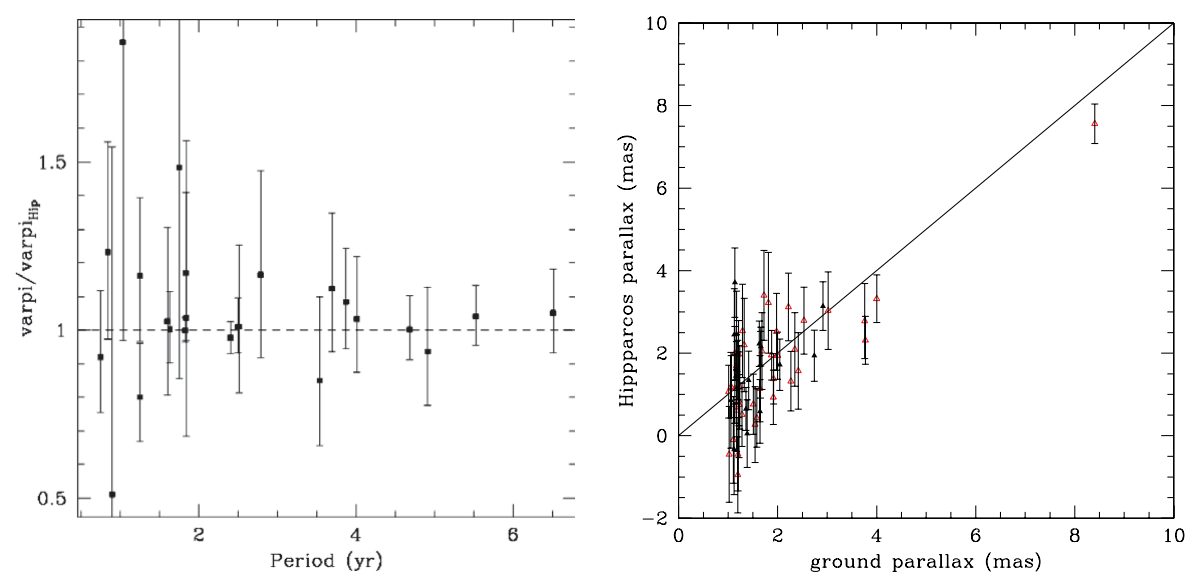

Figure 1. (left) Change of the parallax of binary systems (expressed as the ratio of the parallax derived from the orbital model to the parallax from the single-star model as derived by the Hipparcos team) versus the orbital period (Pourbaix \& Jorissen 2000). (right) Hipparcos Cepheids. Open (filled) triangles denote binary (single) Cepheids.

imposing the single-star model might severely bias the parallax of 1 yr-period binaries, without any chance of detecting the error. According to $S_{B}^{9}$ (Pourbaix et al. 2004), 0.7\% of binaries have a period in the 350-380 day range, but the exact percentage for systems characterized by orbital periods close to one year is difficult to derive observationally.

\section{Blind analysis}

To assess the effect of binarity on the derived parallaxes, a Universe of $9.5 \times 10^{6}$ objects was simulated, with approximately half consisting of genuine multiple stars on average (Arenou 2011). That Universe was subsequently observed with a Gaia simulator in order to produce a realistic end-of-mission time series of positions. These artificial observations were then used to derive the parallaxes, assuming the single-star model for every object, and compared with the true parallaxes. For lots of distant objects, the derived parallax departs from reality (see Fig. 2, left).

In the Hipparcos era, objects with a poor single-star solution were reprocessed using a succession of increasingly sophisticated astrometric models (acceleration, variability induced mover or orbital). For Gaia, the set of possible models will be expanded, especially those including a component of variable brightness. However, owing to the large number of objects that must be processed, the selection of the appropriate model for a given object has to be fully automated and robust (as regards Hipparcos, the approach was sometimes dictated by the known nature of the object). The adopted approach consists of evaluating the models with increasing complexity (roughly speaking, the number of parameters) until a model yields a satisfactory fit. By evaluating the models with increasing complexity, one makes sure that the simplest satisfactory model is selected at the lowest cost in terms of computing resources.

One could nevertheless argue that by selecting the simplest model, one does not benefit from the full potential of the data. This would be true. However, it also prevents overly ambitious models from being adopted (Han et al. 2001). Adopting the orbital model (and leaving all parameters free) when no binarity signature is present would lead to an orbit with a period of one year and a semi-major axis which would be completely correlated with the parallax. Fig. 2 (right) confirms that even if a Keplerian ellipse that is only 

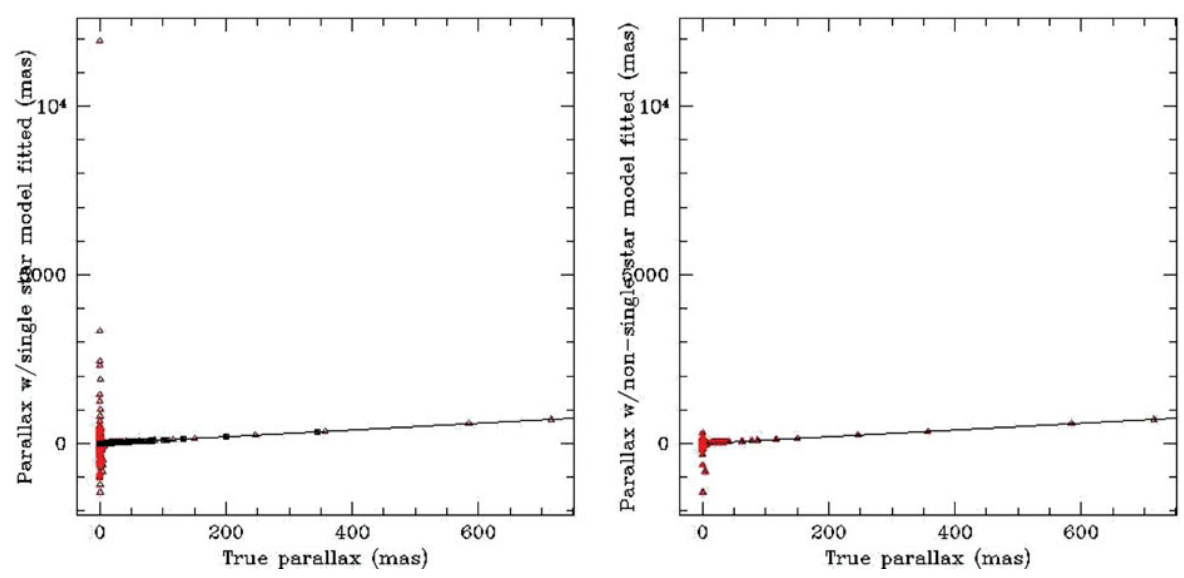

Figure 2. (left) Parallaxes from a single-star model fit. Open triangles denote fits of poor quality (i.e. normalised $\chi^{2}>3$ ). (right) Revised parallaxes for the non-single-star model. Only the previously poorly fitted open triangles are shown.

partially observed is approximated by an arc of a parabola, the parallax associated with this simple model is usually consistent with reality.

\section{Negative parallaxes}

None of the possible models (including the single-star model) imposes a positive parallax. At low signal-to-noise ratios, it is therefore likely that some negative values will occur. Approximately $7 \%$ of the adopted single-star solutions have negative parallaxes. However, only $0.05 \%$ are negative at the $3 \sigma$ level, which is slightly below the expected fraction $(0.135 \%)$. For more sophisticated models, only $2.3 \%$ of the derived parallaxes are negative but of order $1 \%$ remain significant at the $3 \sigma$ level. That $1 \%$ level remains unchanged no matter which one of the sophisticated models is adopted.

Two models are robust as regards their contribution to negative parallaxes. The VIMF model (Variability Induced Mover with a Fixed relative position of the secondary, which was simply called 'VIM' in the Hipparcos data reduction) corresponds to a fixed configuration (the orbital period is much longer than the mission's duration) with one component of variable brightness. The stochastic model is the ultimate model which may be adopted if nothing else fits the data. It is essentially the single-star model with an adjusted noise level. These two models account for respectively 16 and $8 \%$ of the sophisticated solutions. However, they account for respectively 57 and $20 \%$ of the negative parallaxes (see Fig. 3 , middle and right-hand panels). The left-hand panel of Fig. 3 summarises all the other models. The reason for this behaviour, especially for the VIMF, is still under investigation.

\section{Conclusion}

Although exclusive use of the single-star model can severely bias parallax determinations in case of genuine multiple systems, we have shown that a cascade of models with increasing complexity largely improves the reliability of the parallaxes. However, a significant fraction of negative parallaxes is still found among the VIMF and stochastic solutions; regarding the latter, this is not surprising since they are derived from a slightly modified single-star model. Moreover, the parallax of systems with periods close to one year remains uncertain, owing to correlation with the semi-major axis. The proposed 


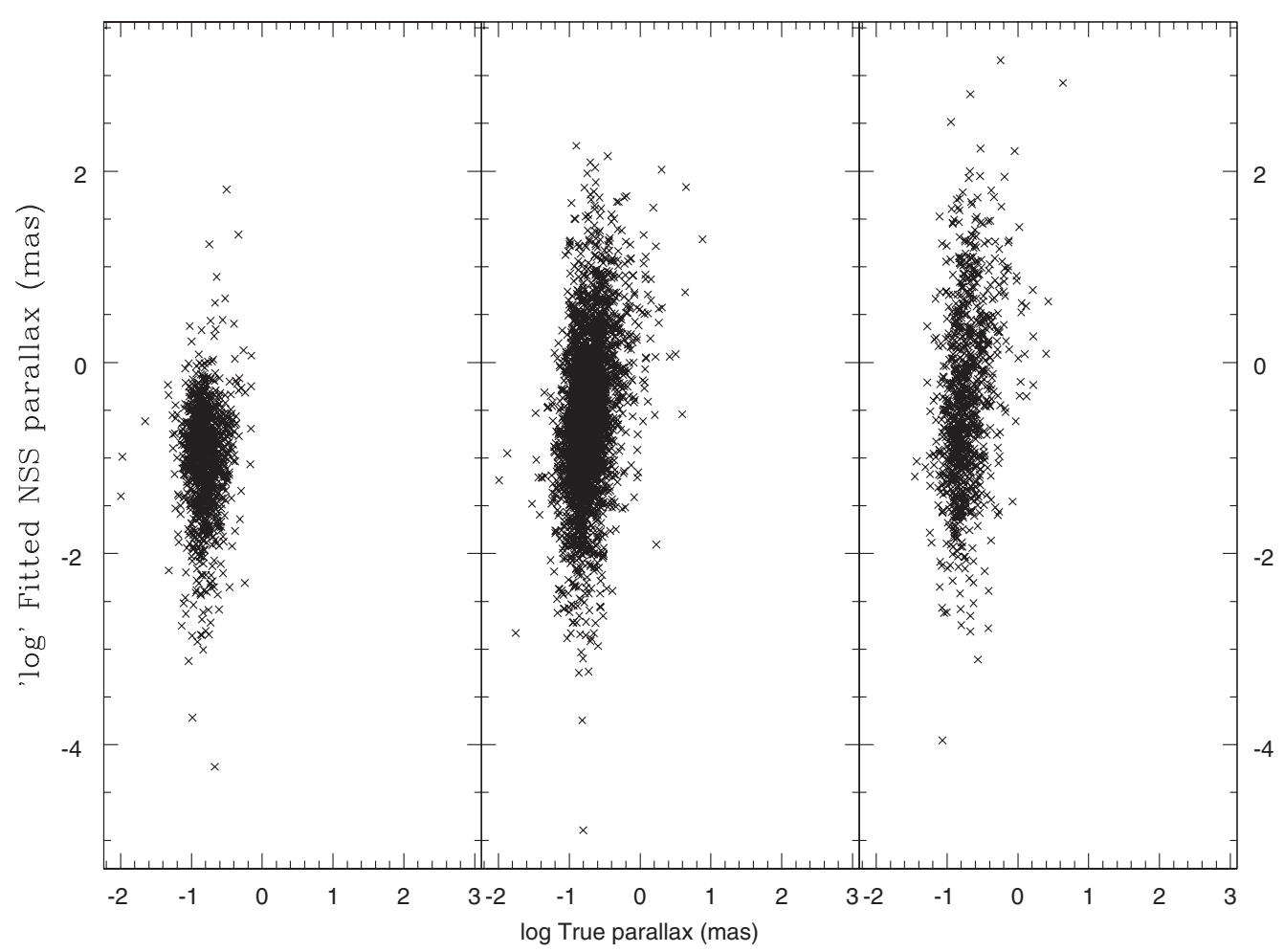

Figure 3. (left) Negative parallaxes from models which are neither VIMF (Variability Induced Mover with a Fixed position angle of the secondary) nor stochastic. (middle) Negative parallaxes from VIMF solutions. (right) Negative parallaxes from stochastic solutions. For each of the three panels, the $x$ axis represents the base- 10 logarithm of the absolute value of the parallax.

processing scheme does not depend on the nature of the object, thus protecting standard candles from any systematic bias caused by the data processing.

\section{Acknowledgements}

DP and CS are supported by an ESA PRODEX research grant.

\section{References}

Arenou F. 2011, in: International Workshop on Double and Multiple Stars: Dynamics, Physics, and Instrumentation (Docobo, J. A., Tamazian, V. S., \& Balega, Y. Y., eds.): AIP, p. 107 ESA 1997, The Hipparcos and Tycho Catalogues, ESA SP-1200

Han, I., Black, D. C., \& Gatewood G. 2001, ApJ, 548, L57

Pourbaix, D. \& Jorissen, A. 2000, A\&AS, 145, 161

Pourbaix, D., Tokovinin, A. A., Batten, A. H., et al. 2004, A\&A A, 424, 727

Szabados L., 1997, in: Hipparcos - Venice 'g7' (Bonnet, R., Høg, E., Bernacca, P.L., et al., eds.), ESA SP-402, p. 657 